\title{
Identifying suitable substrates for high-quality graphene-based heterostructures L. Banszerus,${ }^{\dagger}$ H. Janssen,${ }^{\dagger}$ M. Otto,$\stackrel{ }{\ddagger}$ A. Epping $,{ }^{\dagger}, \mathbb{I}$ T. Taniguchi, ${ }^{\S}$ K. Watanabe,${ }^{\S}$ B. Beschoten, ${ }^{\dagger}$ D. Neumaier, ${ }^{\ddagger}$ and C. Stampfer, ${ }^{*},$, II \\ JARA-FIT and 2nd Institute of Physics, RWTH Aachen University, 52074 Aachen, Germany, Advanced Microelectronic Center Aachen (AMICA), AMO GmbH, Otto-Blumenthal-Straße 25, 52074 Aachen, Germany, Peter Grünberg Institute (PGI-9), Forschungszentrum Jülich, 52425 Jülich, Germany, and National Institute for Materials Science, 1-1 Namiki, Tsukuba 305-0044, Japan \\ Received October 28, 2016; E-mail: stampfer@physik.rwth-aachen.de
}

\begin{abstract}
We report on a scanning confocal Raman spectroscopy study investigating the strain-uniformity and the overall strain and doping of high-quality chemical vapour deposited (CVD) graphenebased heterostuctures on a large number of different substrate materials, including hexagonal boron nitride ( $\mathrm{hBN}$ ), transition metal dichalcogenides, silicon, different oxides and nitrides, as well as polymers. By applying a hBN-assisted, contamination free, dry transfer process for CVD graphene, high-quality heterostructures with low doping densities and low strain variations are assembled. The Raman spectra of these pristine heterostructures are sensitive to substrate-induced doping and strain variations and are thus used to probe the suitability of the substrate material for potential high-quality graphene devices. We find that the flatness of the substrate material is a key figure for gaining, or preserving high-quality graphene.
\end{abstract}

\section{Introduction}

For over a decade, graphene has been in the spotlight of solid state research. Its high charge carrier mobilities $1-4$ and long spin diffusion lengths, ${ }^{5,6}$ as well as its optical ${ }^{7}$ and mechanical properties $\frac{8}{8}$ promise a wide range of applications ranging from spintronics 9 to high frequency electronics, $\frac{10}{}$ ultra-sensitive sensors 11,12 and flexible optoelectronics. 13 In order to advance prototype devices to true applications, large effort has been put into growth ${ }^{14}-18$ and contamination-free transfer $3,4,19,20$ of high quality graphene based on chemical vapour deposition. However, as graphene and other two-dimensional (2d) materials consist only of surface atoms, the choice of substrate material has a large influence on their structural and electronic properties. 2,21-25 In this work, we investigate strain, doping and the strain uniformity of high quality CVD graphene/hBN heterostructures placed on different substrate materials. Here, we follow a recently reported, contamination free, dry transfer process, where exfoliated $\mathrm{hBN}$ is used to pick up CVD graphene directly from the growth substrate. The obtained stack is subsequently placed on different target substrates. $\stackrel{4}{-}$ This fabrication process yields high quality heterostructures with little intrinsic overall doping and low nanometre-scale strain variations. As the graphene crystal is covered, i.e. protected by $\mathrm{hBN}$ on the top side, modifications in doping and strain are purely due to the substrate at the bottom side of graphene, making our approach suitable for benchmarking the substrate suitability.

\footnotetext{
$\dagger$ JARA-FIT and 2nd Institute of Physics, RWTH Aachen University, 52074 Aachen, Germany

$¥$ Advanced Microelectronic Center Aachen (AMICA), AMO GmbH, OttoBlumenthal-Straße 25, 52074 Aachen, Germany

IPeter Grünberg Institute (PGI-9), Forschungszentrum Jülich, 52425 Jülich, Germany

${ }^{\S}$ National Institute for Materials Science, 1-1 Namiki, Tsukuba 305-0044, Japan
}
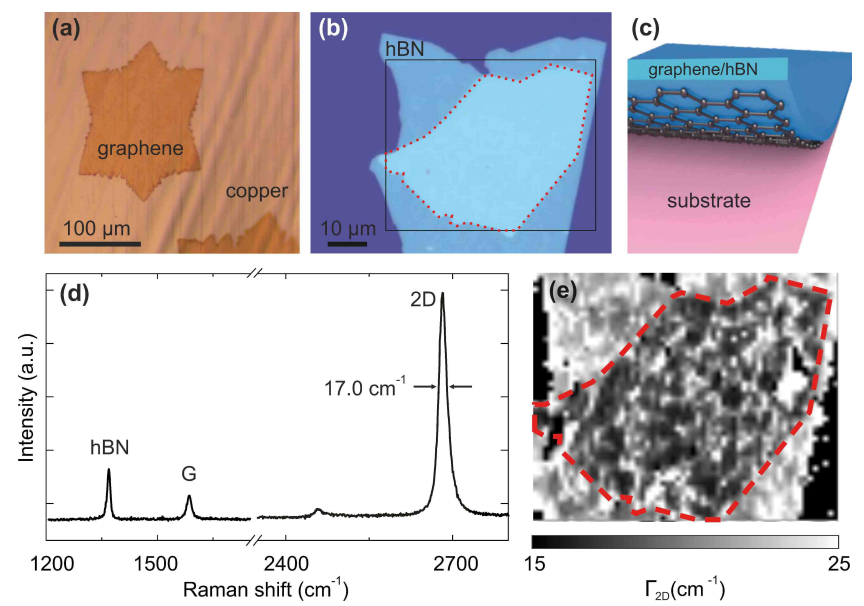

Figure 1. (a) Optical image of a graphene crystal on copper foil a few days after growth. The orange colour is due to the oxidised interface between graphene and the subjacent copper. (b) Optical microscope image of a hBN/CVD-graphene/hBN heterostructure. The black rectangle marks the area that has been mapped using confocal Raman microscopy. The red dashed line marks the sandwiched area. (c) Schematic of a hBN/graphene/substrate structure. (d) Typical Raman spectrum of CVD graphene encapsulated between two flakes of hBN. (e) Raman map of the line width of the Raman 2D-peak, $\Gamma_{2 \mathrm{D}}$ of the area marked in panel (b).

\section{Sample fabrication}

We grow individual graphene crystals with a typical diameter of a few hundred micrometers using a low pressure chemical vapour deposition process at a growth temperature of $1035^{\circ} \mathrm{C}$ on the inside of enclosures folded from commercial copper foil (AlfaAesar 46365). Growth is carried out in a hydrogen/methane atmosphere (45 sccm and $5 \mathrm{sccm}$ respectively) at a total pressure of $0.1 \mathrm{mbar}$ for two hours. 14 Similar to previous works, we let the interface between graphene and copper oxidise under ambient conditions for a few days prior to transfer, in order to weaken the adhesion of the graphene to the copper foil. $\frac{3,4}{4}$ Figure 1(a) shows an optical microscope image of an individual graphene crystal a few days after growth. The bright orange colour is caused by the copper oxide layer on the copper-to-graphene interface. In order to transfer the graphene from the copper foil onto a different substrate, we employ a contamination-free van-der-Waals dry-transfer process. For the transfer, a polymer stamp consisting of a polydimethylsiloxane (PDMS) cushion is covered with a polyvinylalcohol (PVA)/ polymethylmethacrylate (PMMA) stack with a flake of exfoliated hBN on top. The hBN flake is aligned with the graphene and brought into contact at a temperature of $125^{\circ} \mathrm{C}$ and separated again from the copper foil. Subsequently, the graphene/hBN stack is placed on different target substrates and the polymers are dissolved in 

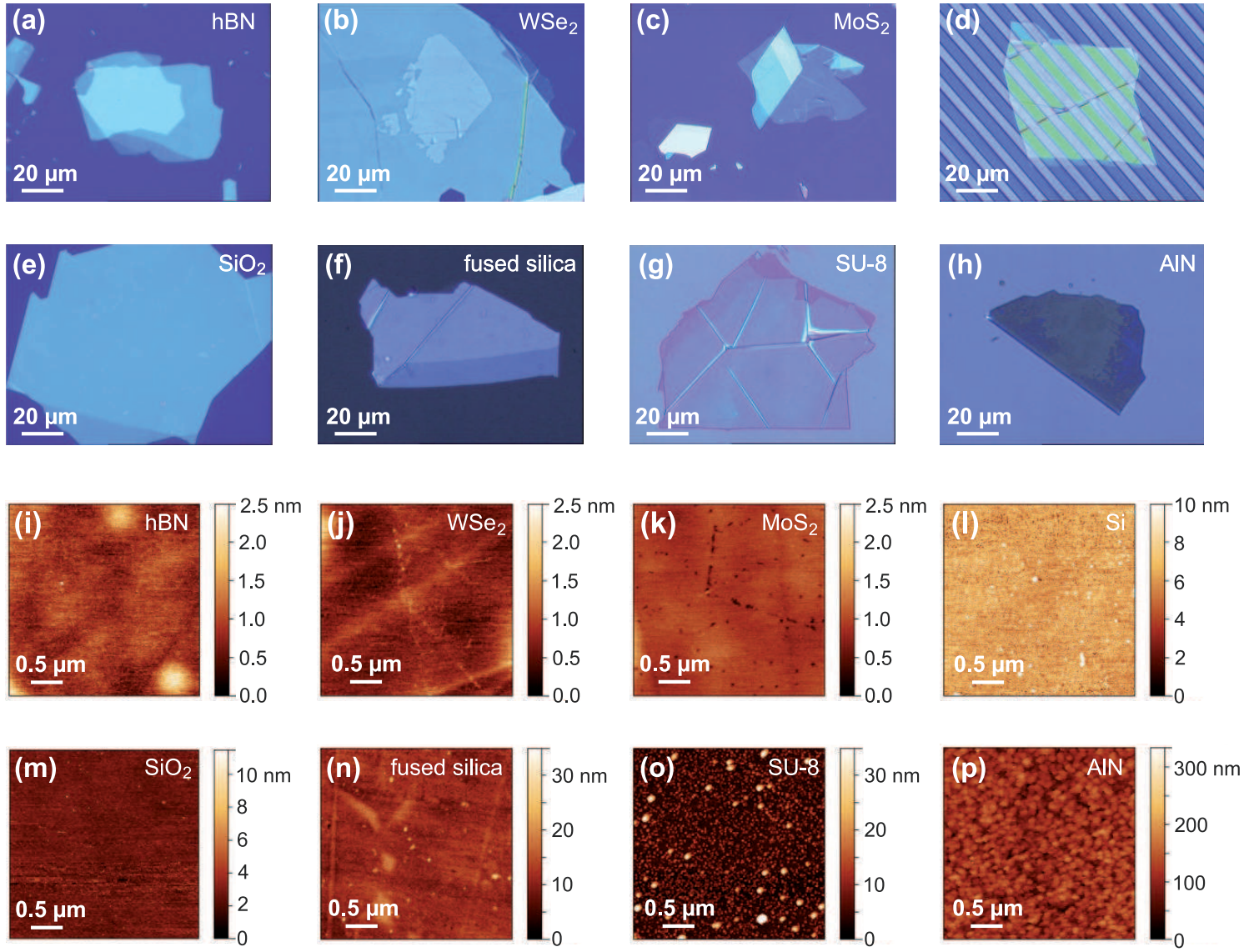

Figure 2. Optical microscope images of the hBN/graphene/substrate heterostructures based on different substrates including (a) hBN, (b) $\mathrm{WSe}_{2}$, (c) $\mathrm{MoS}_{2}$, (d) graphene $\mathrm{hBN}$ suspended over trenches, (e) $\mathrm{Si}^{++} / \mathrm{SiO}_{2}$, (f) fused Silica (g) SU-8, and (h) aluminum nitride (AlN). Atomic force microscopy images of the substrate materials including (i) $\mathrm{hBN}$, (j) $\mathrm{WSe}_{2}$, (k) $\mathrm{MoS}_{2}$, (l) Silicon, (m) $\mathrm{Si}^{++} / \mathrm{SiO}_{2}$, (n) fused Silica (o) SU-8, and (p) aluminium nitride (AlN).

deionized-water, acetone and isopropanol.

\section{Results and discussion}

Figure 1(b) shows an optical microscope image of a graphene/hBN stack placed on another flake of hBN. In figure $1(\mathrm{c})$, we illustrate a schematic of such a hBN/graphene stack on an arbitrary substrate. In previous works, we reported on very little overall doping concentrations $\left(n<3 \times 10^{11} \mathrm{~cm}^{-2}\right)$ and charge carrier mobilities in the range of millions of $\mathrm{cm}^{2} /(\mathrm{Vs})$ for similar $\mathrm{hBN} / \mathrm{graphene} / \mathrm{hBN}$ structures,,$\frac{3}{3}$ indicating that the transfer process avoids degradations of the graphene quality. ${ }^{3.4}$ In order to investigate the quality of the resulting substrate/graphene/hBN stacks, we use scanning confocal Raman microscopy, which is a fast and non-invasive method for probing a number of material properties of graphene including strain, doping, defects and nanometre-scale strain variations. $26-32$ Confocal Raman microscopy was carried out using a WiTec 300 system using a laser with $532 \mathrm{~nm}$ wavelength and a typical laser power of $1 \mathrm{~mW}$. Regions with cracks and folds in the hBN are disregarded in the analysis. Figure 1(d) shows a typical Raman spectrum of graphene sandwiched between two flakes of hBN. The Raman peak originating from the $\mathrm{hBN}$ is located around $\omega_{\mathrm{hBN}}=1365 \mathrm{~cm}^{-1}$. The absence of the Raman D-peak at $1345 \mathrm{~cm}^{-1}$ indicates a very low defect density in the transferred CVD graphene. The Raman G-peak located around $\omega_{\mathrm{G}}=1582 \mathrm{~cm}^{-1}$ shows a full-width-at-half-maximum (FWHM), $\Gamma_{\mathrm{G}}$, of around $14.5 \mathrm{~cm}^{-1}$, indicating very little overall doping of the graphene-based heterostructure. ${ }^{33}$ The Raman 2D-peak is located around $\omega_{2 \mathrm{D}}=2678 \mathrm{~cm}^{-1}$. The FWHM of the 2D-peak, $\Gamma_{2 \mathrm{D}}$, is $17 \mathrm{~cm}^{-1}$ indicating a high sample quality. $\stackrel{4}{-}$ Recently, it has been shown that the value of $\Gamma_{2 \mathrm{D}}$ is related to the amount of nanometrescale strain variations in the graphene lattice within the spot size of the laser. 31 This is particularly crucial as there are strong indications that nanometre-scale strain variations are a limiting factor for the charge carrier mobility in high-quality graphene. ${ }^{25}$ This stresses the importance of using the line width of the Raman 2Dpeak in order to characterise potential substrates for graphene. Figure 1(e) shows a Raman map of $\Gamma_{2 D}$ recorded in the area marked by the black box in figure $1(\mathrm{~b})$. Here, $\Gamma_{2 \mathrm{D}}$ is homogeneously distributed around $18 \mathrm{~cm}^{-1}$ over the entire sandwiched region of the sample [see red dashed line in figures 1(b) and 1(e)], indicating a homogeneous sample quality with little strain variations over the entire sandwiched area. Outside the sandwiched area where the $\mathrm{hBN} /$ graphene stack lays on $\mathrm{SiO}_{2}, \Gamma_{2}$ D shows elevated values of around $23 \mathrm{~cm}^{-1}$, revealing substrate-induced strain variations in the graphene.

The data extracted from the collected Raman spectra on the CVD graphene/hBN sandwich area are very similar to those of high quality heterostructures obtained with exfoliated graphene, $2,24,31$ showing that neither the CVD growth nor our dry transfer method are limiting factors for the 'structural' quality (i.e. overall, 'microscopic' strain and nanometre-scale strain variations) and doping of the graphene-based heterostructures.

In order to investigate and identify suitable other (potentially more scalable) substrate materials, we fabricate stacks of graphene/hBN heterostructures and place them on a number of different substrates. Figures 2(a)-2(h) show optical images of graphene/hBN stacks on such different substrate materials. The substrates investigated in this study are $\mathrm{hBN}$, transition metal 

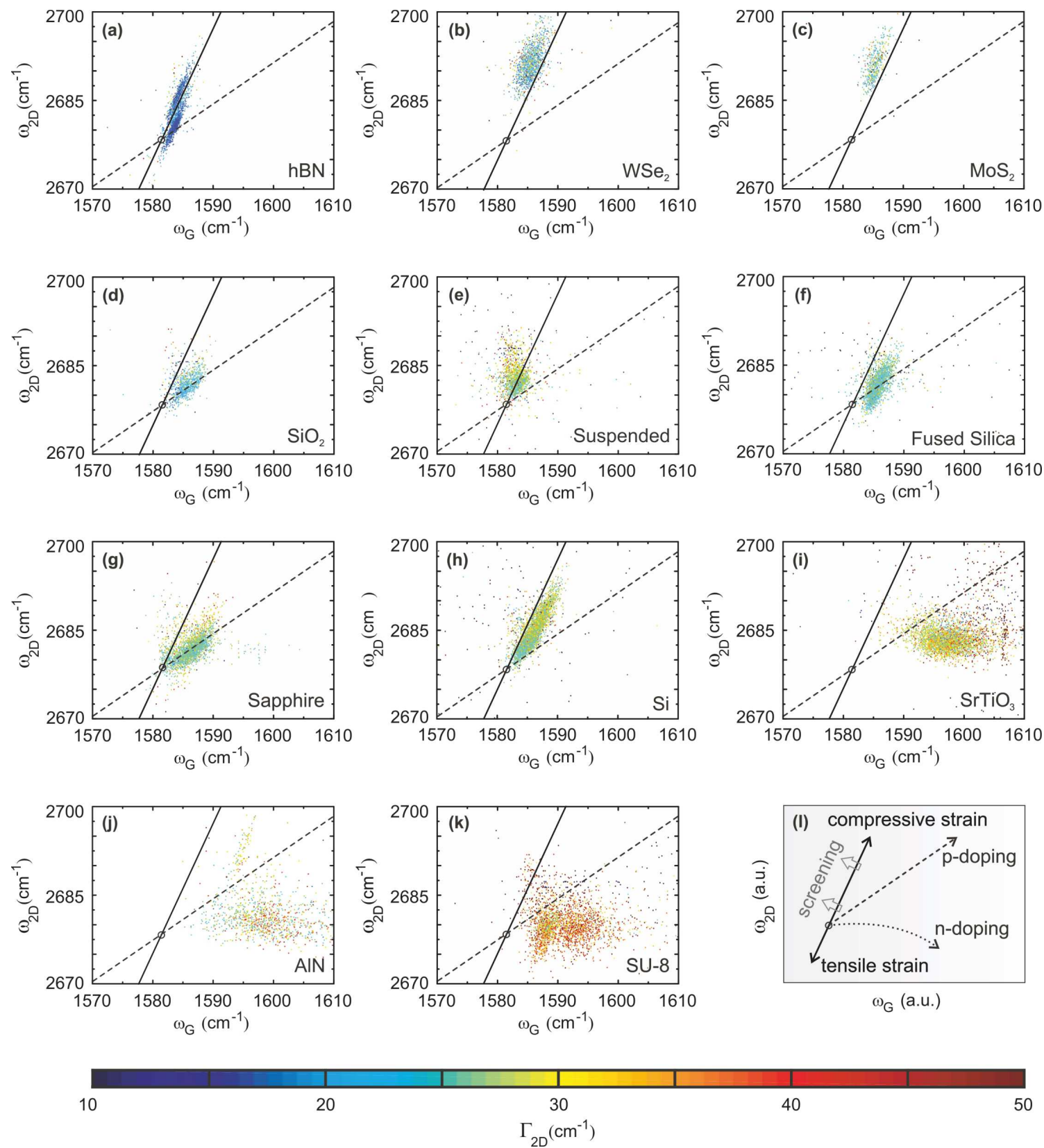

Figure 3. (a)-(k) Scatter plots of $\omega_{2 \mathrm{D}}$ vs. $\omega_{\mathrm{G}}$ extracted from the spatially resolved Raman maps recorded on different substrates. Strain and doping of the graphene can be extracted using a kind of vector decomposition model. 32 The black dashed lines correspond to the doping axes and the black solid lines correspond to the strain axes. The black circles represent the point of pristine unstrained graphene. (1) Schematic representation of the influence of strain, pand n-doping, as well as dielectric screening on $\omega_{2 \mathrm{D}}$ and $\omega_{\mathrm{G}}$.

dichalcogenides (TMDCs) such as $\mathrm{WSe}_{2}$ and $\mathrm{MoS}_{2}, \mathrm{Si}^{++} / \mathrm{SiO}_{2}$, sapphire, $\mathrm{SrTiO}_{3}$, graphene/hBN suspended over trenches, silicon, hydrogen-terminated fused silica, aluminium nitride (AlN) and cross-linked SU-8 (negative epoxy resist from MicroChem). These substrates cover the material classes of oxides, nitrides, silicon, polymers and 2d-materials. With this selected set of substrate materials, we investigate in particular technologically relevant substrate materials as well as upcoming $2 \mathrm{~d}$ materials, which have already been shown to be good and potentially scalable substrates for graphene. ${ }^{22}$ In order to understand the influence of the surface roughness of the substrate on the structural properties of graphene, the substrate roughness is measured using atomic force microscopy (AFM). Figures 2(i)-2(p) show AFM images of the different substrates used. The AFM maps are used to extract the root mean square (RMS) values of the surface roughness for the individual substrate materials. The 2d-materials $\mathrm{hBN}, \mathrm{WSe}_{2}$ and $\mathrm{MoS}_{2}$ show the smallest roughness with RMS values less than $0.2 \mathrm{~nm}$. Silicon, sapphire and $\mathrm{Si}^{++} / \mathrm{SiO}_{2}$ show RMS values between $0.5 \mathrm{~nm}$ and $1 \mathrm{~nm}$. Hydrogen-terminated fused silica, $\mathrm{SrTiO}_{3}$ and $\mathrm{SU}-8$ have a roughness between $2 \mathrm{~nm}$ and $4 \mathrm{~nm}$ and the AlN substrate has the largest roughness with an RMS value of $26 \mathrm{~nm}$. Kretinin and co-workers $^{22}$ report RMS values of $0.1 \mathrm{~nm}$ for $\mathrm{hBN}$ and TMDCs and around $1 \mathrm{~nm}$ for the oxides they investigated, which compares well with the values we observe. Independently, it is important to note that the measured RMS roughness values are not intrinsic substrate properties, but are generally influenced by the used deposition method and subsequent substrate treatments.

In figure 3, we summarize all the information about substrateinduced strain, doping and nanometre-scale strain variations obtained from the Raman maps of graphene/hBN on each substrate 


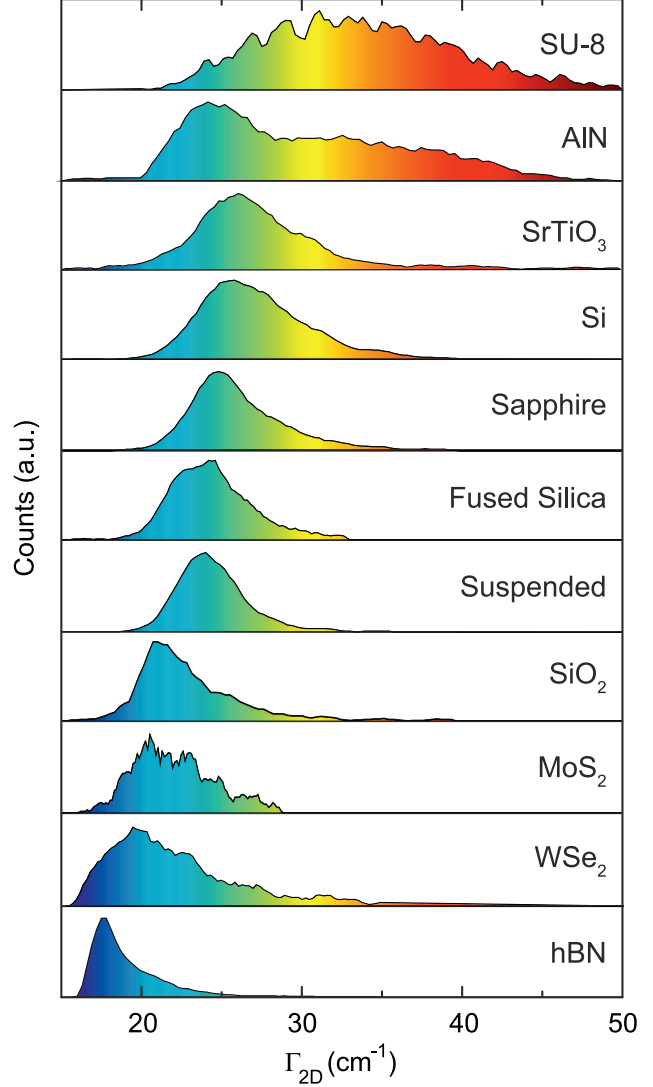

Figure 4. Histograms of $\Gamma_{2 D}$ values extracted from the Raman maps taken on the graphene/hBN heterostructures placed on the different substrates investigated. The colour code is adapted from figure 3 .

material by showing scatter plots of $\omega_{2 \mathrm{D}}$ and $\omega_{\mathrm{G}}$. The amount of nanometre-scale strain variations is linked to $\Gamma_{2 \mathrm{D}}$, which is colour coded in the data points in each panel. In particular, we observe that graphene on $\mathrm{hBN}, \mathrm{WSe}_{2}, \mathrm{MoS}_{2}$, and $\mathrm{SiO}_{2}$ shows low nanometre-scale strain variations as seen by the small values of $\Gamma_{2 \mathrm{D}}$ (blue colour of data points), whereas substrates like $\mathrm{SrTiO}_{3}$, AlN and SU-8 show very large values of $\Gamma_{2 D}$. Besides nanometrescale strain variations, the overall doping (which also is connected to doping variations) strongly influences the transport properties of graphene. In order to compare the amount of substrate-induced strain and doping in graphene for the different substrates, we follow the method reported by Lee et al. ${ }^{32}$ and employ a 'vector' decomposition model on the position of the G-peak and the 2D-peak. A schematic of the influence of strain, doping and dielectric screening of the substrate is depicted in figure 3(1). Strain shifts the 2D-peak and G-peak positions with a relative slope of 2.2 , which is related to the ratio of the Grüneisen parameters for the 2D-peak and the Gpeak phonons 31,32 [see black solid line in figure $3(1)$ and all other panels of figure 3]. Hole doping shifts $\omega_{2 \mathrm{D}}$ and $\omega_{\mathrm{G}}$ with a relative slope of 0.7 [black dashed line in figure 3(1) and all other panels of figure 3] and n-doping results in a non-linear decrease of $\omega_{2 D^{32,34}}$ [see black dotted line in figure 3(1)]. The point of zero strain and zero doping is marked by the black circle. We applied a method by Berciaud et al. $\frac{35}{2}$ and Lee et al. $\frac{32}{}$ in order to determine this point by measuring Raman spectra of pristine suspended graphene. The graphene was in this case directly exfoliated onto a $\mathrm{Si} / \mathrm{SiO}_{2}$ chip with holes etched into the substrate (sample not shown). The G-peak and the 2D-peak of this pristine graphene sample are located at $\omega_{\mathrm{G}}=1581.6 \pm 1 \mathrm{~cm}^{-1}$ and $\omega_{2 \mathrm{D}}=2678.6 \pm 1 \mathrm{~cm}^{-1}$, respectively. In addition to strain and doping, dielectric screening of the substrate material has been shown to shift the peak positions

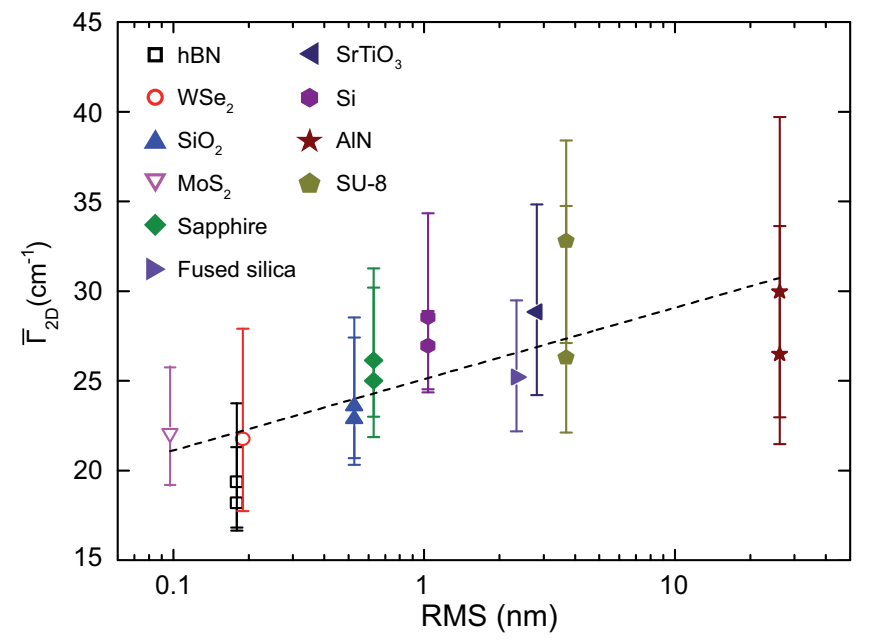

Figure 5. $\quad \bar{\Gamma}_{2 \mathrm{D}}$ versus substrate roughness. The open data points mark the $2 \mathrm{~d}$-layered materials that show self cleaning properties. The black line is a guide to the eye, indicating an increasing $\Gamma_{2 D}$ for increasing surface roughness.

of the G-peak and the 2D-peak as represented by the arrows in figure $3(1)$. 30

Now we have everything to read the information encoded in figures 3(a)-(k). We first compare how far the data points are shifted from the strain axis (black solid line). In particular offsets to the right indicate a minor electronic quality as they are caused by significant doping and doping variations leading to an increased Coulomb scattering. In contrast, small offsets to the left of the black solid line are caused by substrate-induced screening effects, not affecting the (electronic) quality of graphene. All this allows to interpret our data, leading e.g. to the conclusion that $\mathrm{WSe}_{2}$ and $\mathrm{MoS}_{2}$ (only left shifts) are better substrates than $\mathrm{SiO}_{2}$ or sapphire (doping present), even though both substrate classes show almost the same amount of nanometre-scale strain variations. Furthermore, it becomes obvious that $\mathrm{SrTiO}_{3}, \mathrm{AlN}$ and SU-8 not only introduce significant nanometre-scale strain variations, but also lead to substantial charge carrier doping in graphene, making these materials not very suitable for high-quality graphene devices. Notably, the graphene suspended over trenches shows a slightly broadened distribution of $\omega_{2 \mathrm{D}}$ and $\omega_{\mathrm{G}}$ and elevated values for $\Gamma_{2 \mathrm{D}}$, which most likely results from an transfer related contaminations, degrading the graphene quality through the open trenches in the substrate.

Figure 4 shows the individual histograms of the $\Gamma_{2 D}$ values extracted from Raman maps of graphene/ $\mathrm{hBN}$ on all substrates investigated. The histograms for $\mathrm{hBN}$ and $\mathrm{WSe}_{2}$ and $\mathrm{MoS}_{2}$ show very low values of $\Gamma_{2 D}$, indicating a very low amount of nanometrescale strain variations, which makes these $2 \mathrm{~d}$ materials suitable substrates for graphene. Interestingly, these materials exhibit a sharp lower cut-off at around $16 \mathrm{~cm}^{-1}$ suggesting this to be the intrinsic, non-broadened line width of the Raman 2D mode for our laser wavelength. In contrast, the oxidic materials such as $\mathrm{SiO}_{2}$, sapphire and $\mathrm{SrTiO}_{3}$ show elevated values of $\Gamma_{2}$. Both, AlN and SU-8 show extremely high values of $\Gamma_{2 D}$ of up to $50 \mathrm{~cm}^{-1}$ in combination with broad distributions suggesting not only a very high amount of nanometre-scale strain variations, but also nonhomogeneous sample properties.

In figure 5 , we plot the sample averaged $\bar{\Gamma}_{2 \mathrm{D}}$ versus the RMS values of the surface roughness of the substrate material to explore 
Table 1. Representation of the surface roughness (RMS), the sample averaged values of the FWHM of the $2 \mathrm{D}$ peak, $\bar{\Gamma}_{2 \mathrm{D}}$ and the mean values for the position of the G-peak $\bar{\omega}_{\mathrm{G}}$ and of the $2 \mathrm{D}$-peak $\bar{\omega}_{2 \mathrm{D}}$ for all substrate materials investigated.

\begin{tabular}{|l||l|l|l|l|}
\hline & $\begin{array}{l}\text { RMS } \\
(\mathrm{nm})\end{array}$ & $\begin{array}{l}\bar{\Gamma}_{2 \mathrm{D}} \\
\left(\mathrm{cm}^{-1}\right)\end{array}$ & $\begin{array}{l}\bar{\omega}_{\mathrm{G}} \\
\left(\mathrm{cm}^{-1}\right)\end{array}$ & $\begin{array}{l}\bar{\omega}_{2 \mathrm{D}} \\
\left(\mathrm{cm}^{-1}\right)\end{array}$ \\
\hline \hline $\mathrm{hBN}$ & 0.17 & 18.2 & 1583.8 & 2682.6 \\
\hline $\mathrm{WSe}_{2}$ & 0.19 & 21.8 & 1587.2 & 2690.9 \\
\hline $\mathrm{MoS}_{2}$ & 0.09 & 22.0 & 1585.1 & 2690.7 \\
\hline $\mathrm{SiO}_{2}$ & 0.53 & 22.8 & 1585.5 & 2682.5 \\
\hline susp. & - & 24.5 & 1584.2 & 2682.3 \\
\hline fus. silica & 2.3 & 25.2 & 1585.6 & 2681.7 \\
\hline sapphire & 0.63 & 26.1 & 1587.1 & 2682.7 \\
\hline silicon & 1.0 & 26.9 & 1583.2 & 2685.9 \\
\hline SrTiO & 2.8 & 28.8 & 1596.9 & 2683.4 \\
\hline AlN & 26.2 & 30.0 & 1600.3 & 2681.7 \\
\hline SU-8 & 3.7 & 33.6 & 1591.2 & 2681.0 \\
\hline
\end{tabular}

how the presence of nanometre-scale strain variations is related to the surface roughness. The error bars plotted in figure 5 indicate the 20th and the 80th percentile of the corresponding distributions of $\Gamma_{2 \mathrm{D}}$. The hollow data points belong to the $2 \mathrm{~d}$ materials, which exhibit a self cleaning effect. ${ }^{22}$ The black dashed line is a guide to the eye, indicating an increase of the sample averaged $\Gamma_{2 D}$ value as function of an increasing RMS value of the surface roughness. This observation suggest that very flat substrate materials induce very little nanometre-scale strain variations in graphene and - as long as they do not induce large doping/doping variations - are well suited as substrate for high mobility graphene. 25 A similar relation between $\bar{\Gamma}_{2 D}$ and the surface roughness has been observed for the case of graphene on metallic substrates. 36 However, in contrast to Zhao et al., ${ }^{36}$ we observe a smaller variation of $\bar{\Gamma}_{2 D}$ as function of substrate roughness, which may result from the fact that the graphene/hBN heterostack is stiffer than a single layer of graphene and is thus less susceptible to strain than a single layer of graphene on a rough substrate.

Table 1 summarizes our findings including typical RMS values of the surface roughness, the sample averaged values of the FWHM of the $2 \mathrm{D}$ peak, $\bar{\Gamma}_{2 \mathrm{D}}$ and the mean values for the position of the G-peak $\bar{\omega}_{\mathrm{G}}$ and of the 2D-peak $\bar{\omega}_{2 \mathrm{D}}$ for all substrate materials investigated.

\section{Conclusion}

In summary, we use spatially-resolved confocal Raman spectroscopy as a fast, non-invasive characterization tool to investigate the suitability of different material classes as substrates for high quality of graphene, while excluding external or transfer related degradations by protecting the graphene with hBN on top. From the Raman spectra, we extract strain, doping and the strain uniformity for the different substrate materials and present the data in colour-coded scatter plots, which allows to easily decide whether a potential substrate material is suitable for high-quality graphene devices. The presented study suggests that $2 \mathrm{~d}$ materials such as $\mathrm{hBN}$, $\mathrm{WSe}_{2}$ and $\mathrm{MoS}_{2}$ are well suited substrates for graphene, as they possess low values of $\Gamma_{2 D}$ and little overall doping. In contrast, oxidic substrates yield moderate values of $\Gamma_{2 D}$ and intermediate levels of charge carrier doping in graphene. These observations are in good agreement with typical results obtained from transport studies in these material systems. ${ }^{22}$ In general, we observe a trend of decreasing strain uniformity with increasing substrate roughness.

\section{Acknowledgement}

We thank C. Neumann for helpful discussions and gratefully acknowledge support by the Helmholtz-Nanoelectronic-Facility
(HNF), the DFG (SPP-1459), the ERC (GA-Nr. 280140), and the EU project Graphene Flagship (contract no. NECT-ICT-696656). Growth of hexagonal boron nitride crystals was supported by the Elemental Strategy Initiative conducted by the MEXT, Japan and JSPS KAKENHI Grant Numbers JP26248061,JP15K21722 and JP25106006.

\section{References}

(1) K.I. Bolotin, K.J. Sikes, Z. Jiang, M. Klima, G. Fudenberg, J. Hone, P. Kim, H.L. Stormer, Ultrahigh electron mobility in suspended graphene. Solid State Comm., 146, 351, (2011).

(2) L. Wang, I. Meric, P. Y. Huang, Q. Gao, Y. Gao, H. Tran, T. Taniguchi, K. Watanabe, L. M. Campos, D. A. Muller, J. Guo, P. Kim, J. Hone, K. L. Shepard, and C. R. Dean, One-Dimensional Electrical Contact to a TwoDimensional Material. Science 342, 614-617, (2013).

(3) L. Banszerus, M. Schmitz, S. Engels, M. Goldsche, K. Watanabe, T Taniguchi, B. Beschoten, and C. Stampfer, Ballistic Transport Exceeding 28 $\mu \mathrm{m}$ in CVD Grown Graphene. Nano Lett. 16, 1387-1391, (2016).

(4) L. Banszerus, M. Schmitz, S. Engels, J. Dauber, M. Oellers, F. Haupt, K. Watanabe, T. Taniguchi, B. Beschoten, C. Stampfer, Ultrahigh-mobility graphene devices from chemical vapor deposition on reusable copper. Sci. Adv. 1, e1500222, (2015).

(5) M. Drögeler, C. Franzen, F. Volmer, T. Pohlmann, L. Banszerus, M. Wolter, K. Watanabe, T. Taniguchi, C. Stampfer, and B. Beschoten, Spin Lifetimes Exceeding $12 \mathrm{~ns}$ in Graphene Nonlocal Spin Valve Devices. Nano Lett. 16 3533-3539, (2016)

(6) J. Ingla-Aynés, M. H. D. Guimarães, R. J. Meijerink, P. J. Zomer, and B. J. van Wees, $24-\mu \mathrm{m}$ spin relaxation length in boron nitride encapsulated bilayer graphene. Phys. Rev. B 92, 201410(R), (2015).

(7) F. Bonaccorso, Z. Sun, T. Hasan and A. C. Ferrari, Graphene photonics and optoelectronics. Nat. Photonics 4, 611-622 (2010).

(8) C. Lee, X. Wei, J. W. Kysar and J. Hone, Measurement of the Elastic Properties and Intrinsic Strength of Monolayer Graphene. Science, 321, 385-388, (2008).

(9) S. Roche, J. Åkerman, B. Beschoten, J.-C. Charlier, M. Chshiev, S.P. Dash, B. Dlubak, J. Fabian, A. Fert, M. Guimarães, F. Guinea, I. Grigorieva, C. Schönenberger, P. Seneor, C. Stampfer, S. O Valenzuela, X. Waintal, B. van Wees, Graphene spintronics: the European Flagship perspective. 2D Materials, 2, 030202, (2015).

(10) D. Neumaier and H. Zirath, High frequency graphene transistors: can a beauty become a cash cow? 2D Materials, 2, 030203, (2015).

(11) J. Dauber, A. A. Sagade, M. Oellers, K. Watanabe, T. Taniguchi, D. Neumaier, and C. Stampfer, Ultra-sensitive Hall sensors based on graphene encapsulated in hexagonal boron nitride. Appl. Phys. Lett. 106, 193501 (2015).

(12) Z. Wang, L. Banszerus, M. Otto, K. Watanabe, T. Taniguchi, C. Stampfer and D. Neumaier, Encapsulated graphene-based Hall sensors on foil with increased sensitivity. Phys. Status Solidi B., doi: 10.1002/pssb.201600224, (2016).

(13) A. C. Ferrari et al., Science and technology roadmap for graphene, related two-dimensional crystals, and hybrid systems, Nanoscale 7, 4598 (2015).

(14) S. Chen, H. Ji, H. Chou, Q. Li, H. Li, J. W. Suk, R. Piner, L. Liao, W. Cai, R. S. Ruoff, Millimeter-size single-crystal graphene by suppressing evaporative loss of $\mathrm{Cu}$ during low pressure chemical vapor deposition. Adv. Mater 25, 2062-2065, (2013).

(15) X. Li, W. Cai, J. An, S. Kim, J. Nah, D. Yang, R. Piner, A. Velamakanni, I. Jung, E. Tutuc, S. K. Banerjee, L. Colombo, R. S. Ruoff, Large-area synthesis of high-quality and uniform graphene films on copper foils. Science 324, 1312, (2009).

(16) S. Bae, H. Kim, Y. Lee, X. Xu, J. S. Park, Y. Zheng, J. Balakrishnan, T. Lei, H. R. Kim, Y. I. Song, Y. J. Kim, K. S. Kim, B. Ozyilmaz, J. H. Ahn, B. H. Hong, S. Iijima, Roll-to-roll production of 30-inch graphene films for transparent electrodes, Nat. Nanotechnol. 5, 574, (2010).

(17) J.-H. Lee et al., Wafer-Scale Growth of Single-Crystal Monolayer Graphene on Reusable Hydrogen-Terminated Germanium. Science, 344, pp. 286-289 (2014).

(18) X. Li, C. W. Magnuson, A. Venugopal, R. M. Tromp, J. B. Hannon, E. M Vogel, L. Colombo, and R. S. Ruoff, Large-Area Graphene Single Crystals Grown by Low-Pressure Chemical Vapor Deposition of Methane on Copper J. Am. Chem. Soc., 133, 2816, (2011).

(19) J. W. Suk, A. Kitt, C. W. Magnuson, Y. Hao, S. Ahmed, J. An, A. K. Swan, B. B. Goldberg, R. S. Ruoff, Transfer of CVD-grown monolayer graphene onto arbitrary substrates. ACS Nano 5, 6916, (2011).

(20) N. Petrone, C. R. Dean, I. Meric, A. M. van der Zande, P. Y. Huang, L.Wang, D. Muller, K. L. Shepard, and J. Hone, Chemical vapor depositionderived graphene with electrical performance of exfoliated graphene. Nano Lett. 12, 2751, (2012).

(21) W. Gannett, W. Regan, K. Watanabe, T. Taniguchi, M. F. Crommie and A Zettl1, Boron nitride substrates for high mobility chemical vapor deposited graphene. Appl. Phys. Lett. 98, 242105 (2011).

(22) A. V. Kretinin, Y. Cao, J. S. Tu, G. L. Yu, R. Jalil, K. S. Novoselov, S. J. Haigh, A. Gholinia, A. Mishchenko, M. Lozada, T. Georgiou, C. R. Woods, F. Withers, P. Blake, G. Eda A. Wirsig, C. Hucho, K. Watanabe, T. Taniguchi, A. K. Geim, and R. V. Gorbachev, Electronic Properties of Graphene Encapsulated with Different Two-Dimensional Atomic Crystals. Nano Lett. 14 3270-3276, (2014).

(23) A. Pirkle, J. Chan, A. Venugopal, D. Hinojos, C. W. Magnuson, S. McDon- 
nell, L. Colombo, E. M. Vogel1, R. S. Ruoff and R. M. Wallace, The effect of chemical residues on the physical and electrical properties of chemical vapor deposited graphene transferred to SiO2. Appl. Phys. Lett. 99, 122108 (2011).

(24) C. R. Dean, A. F. Young, I. Meric, C. Lee, L. Wang, S. Sorgenfrei, K Watanabe, T. Taniguchi, P. Kim, K. L. Shepard, J. Hone, Boron nitride substrates for high-quality graphene electronics. Nat. Nano. 5, 722, (2010).

(25) Nuno J. G. Couto, Davide Costanzo, Stephan Engels, Dong-Keun Ki, Kenji Watanabe, Takashi Taniguchi, Christoph Stampfer, Francisco Guinea, and Alberto F. Morpurgo, Random Strain Fluctuations as Dominant Disorder Source for High-Quality On-Substrate Graphene Devices. Phys. Rev. X 4, 041019, (2014)

(26) A. C. Ferrari, J C. Meyer, V. Scardaci, C. Casiraghi, M. Lazzeri, F. Mauri, S. Piscanec, D. Jiang, K. S. Novoselov, S. Roth, A. K. Geim, Raman spectrum of graphene and graphene layers. Phys. Rev. Lett. 97, 187401, (2006).

(27) A. C. Ferrari and D. M. Basko, Raman spectroscopy as a versatile tool for studying the properties of graphene. Nat. Nano., 8, pp. 235-246, (2013)

(28) D. Graf, F. Molitor, K. Ensslin, C. Stampfer, A. Jungen, C. Hierold, L. Wirtz, Spatially resolved Raman spectroscopy of single- and few-layer graphene. Nano Lett. 7, 238-242, (2007)

(29) L.M. Malard, M.A. Pimenta, G. Dresselhaus, M.S. Dresselhaus, Raman spectroscopy in graphene. Physics Reports, 473, 51-87, (2009).

(30) F. Forster, A. Molina-Sanchez, S. Engels, A. Epping, K. Watanabe, T. Taniguchi, L. Wirtz, C. Stampfer, Dielectric screening of the Kohn anomaly of graphene on hexagonal boron nitride. Phys. Rev. B 88, 085419, (2013).

(31) C. Neumann, S. Reichardt, P. Venezuela, M. Drögeler, L. Banszerus, M. Schmitz, K. Watanabe, T. Taniguchi, F. Mauri, B. Beschoten, S. V. Rotkin, C Stampfer, Raman spectroscopy as probe of nanometer-scale strain variations in graphene. Nature Communications, 6, 8429, (2015).

(32) J. E. Lee, G. Ahn, J. Shim, Y. S. Lee, S. Ryu, Optical separation of mechanical strain from charge doping in graphene. Nat. Commun. 3, 1024 (2012).

(33) C. Casiraghi, S. Pisana, K. Novoselov, A. Geim, and A. Ferrari, Raman fingerprint of charged impurities in graphene. Appl. Phys. Lett. 91, 233108 (2007).

(34) G. Froehlicher and S. Berciaud, Raman spectroscopy of electrochemically gated graphene transistors: Geometrical capacitance, electronphonon, electron-electron, and electron-defect scattering. Phys. Rev. B 91 , 205413,(2015).

(35) S. Berciaud, X. Li, H. Htoon, L.E. Brus, S.K. Doorn and T.F. Heinz, Intrinsic Line Shape of the Raman-2D mode in Freestanding Graphene Monolayers. Nano Lett. 13, 3517 (2013)

(36) Y. Zhao, X. Liu, D. Y. Lei and Y. Chai, Effects of surface roughness of Ag thin films on surface-enhanced Raman spectroscopy of graphene: spatial nonlocality and physisorption strain. Nanoscale, 6, 1311-1317, (2014). 